\title{
New Double Projection Algorithm for Solving Variational Inequalities
}

\begin{abstract}
Lian Zheng
Department of Mathematics and Computer Science, Yangtze Normal University, Fuling, Chongqing 408100, China

Correspondence should be addressed to Lian Zheng; zhenglian66@yahoo.com.cn

Received 7 January 2013; Accepted 20 May 2013

Academic Editor: Zhongxiao Jia

Copyright ( 2013 Lian Zheng. This is an open access article distributed under the Creative Commons Attribution License, which permits unrestricted use, distribution, and reproduction in any medium, provided the original work is properly cited.

We propose a class of new double projection algorithms for solving variational inequality problem, which can be viewed as a framework of the method of Solodov and Svaiter by adopting a class of new hyperplanes. By the separation property of hyperplane, our method is proved to be globally convergent under very mild assumptions. In addition, we propose a modified version of our algorithm that finds a solution of variational inequality which is also a fixed point of a given nonexpansive mapping. If, in addition, a certain local error bound holds, we analyze the convergence rate of the iterative sequence. Numerical experiments prove that our algorithms are efficient.
\end{abstract}

\section{Introduction}

We consider the following variational inequality problem, denoted by $\operatorname{VI}(C, f)$, to find a vector $x^{*} \in C$ such that

$$
\left\langle f\left(x^{*}\right), y-x^{*}\right\rangle \geq 0, \quad \forall y \in C,
$$

where $C$ is a nonempty closed convex set in $\mathbb{R}^{n}, f$ is a continuous mapping from $\mathbb{R}^{n}$ into itself, and $\langle\cdot, \cdot\rangle$ denotes the usual inner product in $\mathbb{R}^{n}$. Let $\operatorname{SOL}(C, f)$ denote the solution set of $\operatorname{VI}(C, f)$, and let $\Pi_{C}$ denote the projection onto $C$. Throughout this paper, we assume that $\operatorname{SOL}(C, f)$ is nonempty and $f$ has the property that

$$
\left\langle f(y), y-x^{*}\right\rangle \geq 0, \quad \forall y \in C \text { and all } x^{*} \in \operatorname{SOL}(C, f) .
$$

The property (2) holds if $f$ is monotone or more generally pseudomonotone on $C$ in the sense of Karamardian [1].

The variational inequality problems have wide applications in reality. In recent years, many numerical algorithms for $\operatorname{VI}(C, f)$ have been proposed. These methods include Newton method, proximal algorithm, projection algorithm, and their variants; see [2, 3]. Among these methods, projection-type method is a simple and an efficient one; the oldest algorithm of this class is the extragradient projection method introduced in [4] and later refined and extended in [5-7].

In 1999, Solodov and Svaiter [8] proposed a hyperplane projection algorithm for solving the $\operatorname{VI}(C, f)$ in Euclidean space, known also as the double projection algorithm due to the fact that one needs to implement double projections in each iteration. One is onto the feasible set $C$, and the other is onto the intersection of the feasible set $C$ and the half-space. More precisely, they presented the following algorithm.

Algorithm 1. Choose an intial point $x^{0}$, parameters $\mu>0$, $\sigma, \gamma \in(0,1)$ and set $k=0$.

Step 1. Having $x^{k}$, compute

$$
z^{k}=\Pi_{C}\left[x^{k}-\mu f\left(x^{k}\right)\right] .
$$

Stop if $x^{k}=z^{k}$; otherwise, go to Step 2.

Step 2. Compute $y^{k}=x^{k}-\eta_{k}\left(x^{k}-z^{k}\right)$, where $\eta_{k}=\gamma^{m_{k}}$ with $m_{k}$ being the smallest nonnegative integer $m$ such that

$$
\left\langle f\left(x^{k}-\gamma^{m}\left(x^{k}-z^{k}\right)\right), x^{k}-z^{k}\right\rangle \geq \sigma\left\|x^{k}-z^{k}\right\|^{2} .
$$

Step 3. Compute

$$
x^{k+1}=\Pi_{C \cap H_{k}}\left(x^{k}\right),
$$


where

$$
H_{k}=\left\{x \in \mathbb{R}^{n} \mid\left\langle f\left(y^{k}\right), x-y^{k}\right\rangle \leq 0\right\} .
$$

Let $k:=k+1$, and return to Step 1 .

Wang et al. [9] shows that Algorithm 1 can get a longer step size, and hence it is a better algorithm than the extragradient method proposed by Korpelevich [7] in theory. The convergence rate of the iterative sequence generated by the hyperplane projection method depends mainly on the choice of hyperplane and projection way. We note that the hyperplane of Algorithm 1 is constructed by $f\left(y^{k}\right)$. In 2006 , He [10] constructed a new hyperplane by a linear combination of $f\left(y^{k}\right)$ and $x^{k}-z^{k}$ and hence modified the Algorithm 1.

Inspired by the above, in this paper, we construct a class of new hyperplanes by a linear combination of $f\left(y^{k}\right), x^{k}-z^{k}$ and $f\left(x^{k}\right)$ and hence present a class of new double projection algorithms. Using the proof method proposed by He in [10], our algorithms are proved to be globally convergent under continuous and pseudomonotone. Numerical experiments show that constructing hyperplane by $f\left(x^{k}\right)$ has a certain significance to change the convergence rate of the iterative sequence. In addition, we propose a modified version of our algorithm that find a solution of variational inequality which is also a fixed point of a given nonexpansive mapping.

The organization of this paper is as follows. In the next section, we give some preliminaries. The details of the double projection algorithm are presented, and its global convergence analysis is proved in Section 3. The modified double projection algorithm and its convergence analysis are in Section 4. Numerical results are reported in Section 5. Finally, conclusions together with some further studies are summarized in the last section.

\section{Preliminaries}

Let $\mu>0$ be a parameter. The natural residual function $r_{\mu}(\cdot)$ is defined by

$$
r_{\mu}(x):=x-\Pi_{C}(x-\mu f(x)) .
$$

A well-known fact is that $x^{*}$ is a solution of $\operatorname{VI}(C, f)$ if and only if $x^{*}$ is a root of $r_{\mu}(\cdot)$.

Lemma 2. Let $C \subset \mathbb{R}^{n}$ be a closed convex set. Then, it holds that

(1) $\left\langle y-\Pi_{C}(y), x-\Pi_{C}(y)\right\rangle \leq 0, \forall x \in C, y \in \mathbb{R}^{n}$,

(2) $\left\|x-\Pi_{C}(y)\right\|^{2} \leq\|x-y\|^{2}-\left\|y-\Pi_{C}(y)\right\|^{2}, \forall x \in C$, $y \in \mathbb{R}^{n}$.

By Lemma 2(1), it is easy to prove that

$$
\left\langle f(x), r_{\mu}(x)\right\rangle \geq \mu^{-1}\left\|r_{\mu}(x)\right\|^{2} .
$$

Lemma 3. For every $x \in C, x^{*} \in \operatorname{SOL}(C, f)$, and $\eta \in[0,1]$, one has

$$
\left\langle x-x^{*}, f\left(x-\eta r_{\mu}(x)\right)\right\rangle \geq \eta\left\langle r_{\mu}(x), f\left(x-\eta r_{\mu}(x)\right)\right\rangle .
$$

Proof. Since $x^{*} \in \operatorname{SOL}(C, f)$, by property (2),

$$
\left\langle f(y), y-x^{*}\right\rangle \geq 0, \quad \forall y \in C .
$$

In inequality (10), substitute $y$ with $x-\eta r_{\mu}(x)$, and we obtain the desired result.

Lemma 4. Let $C$ be a closed convex set in $\mathbb{R}^{n}, h$ be a realvalued function on $\mathbb{R}^{n}$, and $K$ be the set $\{x \in C: h(x) \leq 0\}$. If $K$ is nonempty and $h$ is Lipschitz continuous on $C$ with modulus $\theta>0$, then

$$
\operatorname{dist}(x, K) \geq \theta^{-1} \max \{h(x), 0\}, \quad \forall x \in C,
$$

where dist $(x, K)$ denotes the distance from $x$ to $K$.

Proof. See [10, Lemma 2.3].

Lemma 5. Let $\left\{\alpha_{k}\right\}$ be a real sequence satisfying $0<a \leq \alpha_{k} \leq$ $b<1$ for all $k$, and let $\left\{\nu^{k}\right\}$ and $\left\{\omega^{k}\right\}$ be two sequences in $R^{n}$ such that for some $\theta \geq 0$,

$$
\begin{gathered}
\limsup _{k \rightarrow \infty}\left\|\nu^{k}\right\| \leq \theta, \quad \limsup _{k \rightarrow \infty}\left\|\omega^{k}\right\| \leq \theta, \\
\lim _{k \rightarrow \infty}\left\|\alpha_{k} \nu^{k}+\left(1-\alpha_{k}\right) \omega^{k}\right\|=\theta .
\end{gathered}
$$

Then,

$$
\lim _{k \rightarrow \infty}\left\|\nu^{k}-\omega^{k}\right\|=0
$$

Proof. See [11, Lemma 3.1].

\section{The Double Projection Algorithm and Convergence Analysis}

Algorithm 6. Select $x^{0} \in C, \alpha, \beta \geq 0, \sigma>0, \mu \in\left(0, \sigma^{-1}\right)$, $\gamma \in(0,1), \omega \geq \alpha$. Set $k=0$.

Step 1. For $x^{k} \in C$, define

$$
z^{k}=\Pi_{C}\left(x^{k}-\mu f\left(x^{k}\right)\right) .
$$

Compute $r_{\mu}\left(x^{k}\right)$. If $r_{\mu}\left(x^{k}\right)=0$, stop; else go to Step 2.

Step 2. Compute $y^{k}=x^{k}-\eta_{k} r_{\mu}\left(x^{k}\right)$, where $\eta_{k}=\gamma^{m_{k}}$ with $m_{k}$ being the smallest nonnegative integer $m$ satisfying

$$
\left\langle f\left(x^{k}\right)-f\left(x^{k}-\gamma^{m} r_{\mu}\left(x^{k}\right)\right), r_{\mu}\left(x^{k}\right)\right\rangle \leq \sigma\left\|r_{\mu}\left(x^{k}\right)\right\|^{2} .
$$

Step 3. Compute $x^{k+1}=\Pi_{C_{k}}\left(x^{k}\right)$, where $C_{k}=C \cap H_{k}$ with $H_{k}=\left\{v \in \mathbb{R}^{n}: h_{k}(v) \leq 0\right\}$ being a halfspace defined by the function

$$
h_{k}(v)=\left\langle d^{k}, v-x^{k}\right\rangle+\omega \eta_{k}(1-\mu \sigma)\left\|r_{\mu}\left(x^{k}\right)\right\|^{2},
$$

where normal vector $d^{k}=\alpha \eta_{k} r_{\mu}\left(x^{k}\right)+\beta f\left(x^{k}\right)+\omega \mu f\left(y^{k}\right)$.

Let $k=k+1$, and return to Step 1 . 
Remark 7. In Algorithm 6, the searching direction is taken as

$$
-d^{k}=-\left(\alpha \eta_{k} r_{\mu}\left(x^{k}\right)+\beta f\left(x^{k}\right)+\omega \mu f\left(y^{k}\right)\right)
$$

which is a linear combination set of $r_{\mu}\left(x^{k}\right), f\left(x^{k}\right)$ with $f\left(y^{k}\right)$. When taking $\alpha=1, \beta=0, \omega=\mu^{-1},-d^{k}$ degrade into the direction introduced by $\mathrm{He}$ [10]; when taking $\alpha=\beta=1$, $\omega=\mu^{-1},-d^{k}$ degrade into the direction introduced by Noor et al. [12]; when taking $\alpha=1 / \eta_{k}, \beta=0, \omega=1 / \mu \eta_{k},-d^{k}$ degrade into the direction introduced by Wang et al. [13]; when taking $\alpha=\beta=0, \omega=\mu^{-1},-d^{k}$ degrade into the direction introduced by Iusem and Svaiter [7], Solodov and Svaiter [8], and wang et al. [9], which shows that our direction is a framework of the ones of the above projection methods.

Lemma 8. Let $x^{*} \in \operatorname{SOL}(C, f)$, and let the function $h_{k}$ be defined by (16). Then,

$$
h_{k}\left(x^{k}\right) \geq \omega \eta_{k}(1-\mu \sigma)\left\|r_{\mu}\left(x^{k}\right)\right\|^{2}, \quad h_{k}\left(x^{*}\right) \leq 0 .
$$

In particular, if $r_{\mu}\left(x^{k}\right) \neq 0$, then $h_{k}\left(x^{k}\right)>0$.

Proof. Consider that

$$
\begin{aligned}
h_{k}\left(x^{k}\right) & =\left\langle d^{k}, x^{k}-x^{k}\right\rangle+\omega \eta_{k}(1-\mu \sigma)\left\|r_{\mu}\left(x^{k}\right)\right\|^{2} \\
& =\omega \eta_{k}(1-\mu \sigma)\left\|r_{\mu}\left(x^{k}\right)\right\|^{2} .
\end{aligned}
$$

If $r_{\mu}\left(x^{k}\right) \neq 0$, then $h_{k}\left(x^{k}\right)>0$ because $\mu \sigma<1$. Next, we prove that $h_{k}\left(x^{*}\right) \leq 0$. Since

$$
z^{k}=\Pi_{C}\left(x^{k}-\mu f\left(x^{k}\right)\right)
$$

by (1) of Lemma 2, we have

$$
\left\langle x^{k}-z^{k}-\mu f\left(x^{k}\right), z^{k}-x^{*}\right\rangle \geq 0 .
$$

By property (2),

$$
\left\langle\mu f\left(x^{k}\right), x^{k}-x^{*}\right\rangle \geq 0 .
$$

Adding inequalities (21) and (22), we obtain

$$
\left\langle x^{k}-z^{k}-\mu f\left(x^{k}\right), z^{k}-x^{k}\right\rangle+\left\langle x^{k}-z^{k}, x^{k}-x^{*}\right\rangle \geq 0 .
$$

We have

$$
\begin{aligned}
\left\langle x^{k}-x^{*}, d^{k}\right\rangle & \\
= & \left\langle x^{k}-x^{*}, \alpha \eta_{k} r_{\mu}\left(x^{k}\right)+\beta f\left(x^{k}\right)+\omega \mu f\left(y^{k}\right)\right\rangle \\
= & \alpha \eta_{k}\left\langle x^{k}-x^{*}, x^{k}-z^{k}\right\rangle+\beta\left\langle f\left(x^{k}\right), x^{k}-x^{*}\right\rangle \\
& +\omega \mu\left\langle f\left(y^{k}\right), x^{k}-x^{*}\right\rangle \\
\geq & \alpha \eta_{k}\left\langle r_{\mu}\left(x^{k}\right)-\mu f\left(x^{k}\right), r_{\mu}\left(x^{k}\right)\right\rangle \\
& +\omega \mu \eta_{k}\left\langle f\left(y^{k}\right), r_{\mu}\left(x^{k}\right)\right\rangle \\
= & \alpha \eta_{k}\left\|r_{\mu}\left(x^{k}\right)\right\|^{2}-\alpha \mu \eta_{k}\left\langle f\left(x^{k}\right)-f\left(y^{k}\right), r_{\mu}\left(x^{k}\right)\right\rangle \\
& +(\omega-\alpha) \mu \eta_{k}\left\langle f\left(y^{k}\right), r_{\mu}\left(x^{k}\right)\right\rangle \\
\geq & \alpha \eta_{k}\left\|r_{\mu}\left(x^{k}\right)\right\|^{2}-\alpha \mu \sigma \eta_{k}\left\|r_{\mu}\left(x^{k}\right)\right\|^{2} \\
& +(\omega-\alpha) \mu \eta_{k}\left(\mu{ }^{-1}-\sigma\right)\left\|r_{\mu}\left(x^{k}\right)\right\|^{2} \\
= & \eta_{k} \omega(1-\mu \sigma)\left\|r_{\mu}\left(x^{k}\right)\right\|^{2},
\end{aligned}
$$

where the first inequality follows from (2), (9), and (23) and the last one follows (8) and (15).

It follows that

$$
\begin{aligned}
h_{k} & \left(x^{*}\right) \\
& =\left\langle d^{k}, x^{*}-x^{k}\right\rangle+\eta_{k} \omega(1-\mu \sigma)\left\|r_{\mu}\left(x^{k}\right)\right\|^{2} \\
& =-\left\langle d^{k}, x^{k}-x^{*}\right\rangle+\eta_{k} \omega(1-\mu \sigma)\left\|r_{\mu}\left(x^{k}\right)\right\|^{2} \\
& \leq-\eta_{k} \omega(1-\mu \sigma)\left\|r_{\mu}\left(x^{k}\right)\right\|^{2}+\eta_{k} \omega(1-\mu \sigma)\left\|r_{\mu}\left(x^{k}\right)\right\|^{2} \\
& =0 .
\end{aligned}
$$

The proof is completed.

Now, we turn to consider the convergence of Algorithm 6. Certainly, if Algorithm 6 terminates at Step $k$, then $x^{k}$ is a solution of $\operatorname{VI}(C, f)$. Therefore, in the following analysis, we assume that Algorithm 6 always generates an infinite sequence.

Theorem 9. IfC is a nonempty closed and convex set in $\mathbb{R}^{n}$ and the property (2) holds, then the sequence $\left\{x^{k}\right\} \subset \mathbb{R}^{n}$ generated by Algorithm 6 is bounded and $\lim _{k \rightarrow \infty} \operatorname{dist}\left(x^{k}, C_{k}\right)=0$.

Proof. Let $x^{*} \in \operatorname{SOL}(C, f)$. Since

$$
x^{k+1}=\Pi_{C_{k}}\left(x^{k}\right),
$$


it follows from Lemma 2(2) that

$$
\begin{aligned}
\left\|x^{k+1}-x^{*}\right\|^{2} & \leq\left\|x^{k}-x^{*}\right\|^{2}-\left\|x^{k+1}-x^{k}\right\|^{2} \\
& =\left\|x^{k}-x^{*}\right\|^{2}-\operatorname{dist}^{2}\left(x^{k}, C_{k}\right) .
\end{aligned}
$$

It follows that the sequence $\left\{\left\|x^{k+1}-x^{*}\right\|^{2}\right\}$ is nonincreasing, and hence it is a convergent sequence. Therefore, $\left\{x^{k}\right\}$ is bounded and $\lim _{k \rightarrow \infty} \operatorname{dist}\left(x^{k}, C_{k}\right)=0$. The proof is completed.

Theorem 10. If $C$ is a nonempty closed and convex set in $\mathbb{R}^{n}$, $f$ is a continuous mapping from $\mathbb{R}^{n}$ into itself, and the property (2) holds, then Algorithm 6 generates an infinite sequence $\left\{x^{k}\right\}$ converging to a solution of $\operatorname{VI}(C, f)$.

Proof. By Theorem 9, the sequence $\left\{x^{k}\right\}$ is bounded. Since $f$ and projection operator are continuous, we have the sequence $\left\{z^{k}\right\}$, and hence the sequence $\left\{y^{k}\right\}$ is bounded. Thus, $\left\{f\left(x^{k}\right)\right\}$, $\left\{f\left(z^{k}\right)\right\}$, and $\left\{r_{\mu}\left(x^{k}\right)\right\}$ are bounded sequences; that is, there exists some $M>0$, such that

$$
\left\|d^{k}\right\|=\left\|\alpha \eta_{k} r_{\mu}\left(x^{k}\right)+\beta f\left(x^{k}\right)+\omega \mu f\left(y^{k}\right)\right\| \leq M, \quad \forall k .
$$

Clearly each function $h_{k}$ is Lipschitz continuous on $C$ with modulus $M$. By Lemmas 8 and 4, we obtain that

$$
\operatorname{dist}\left(x^{k}, C_{k}\right) \geq M^{-1} h_{k}\left(x^{k}\right) \geq M^{-1} \omega \eta_{k}(1-\mu \sigma)\left\|r_{\mu}\left(x^{k}\right)\right\|^{2} \text {. }
$$

Thus, by Theorem 9, we have

$$
\lim _{k \rightarrow \infty} \eta_{k}\left\|r_{\mu}\left(x^{k}\right)\right\|^{2}=0
$$

which implies that there exist subsequences $\left\{x^{k_{i}}\right\}$ and $\left\{x^{k_{j}}\right\}$ of $\left\{x^{k}\right\}$, respectively, such that

$$
\lim _{i \rightarrow \infty}\left\|r_{\mu}\left(x^{k_{i}}\right)\right\|=0, \quad \text { or } \quad \lim _{j \rightarrow \infty} \eta_{k_{j}}=0 .
$$

Suppose that $\lim _{i \rightarrow \infty}\left\|r_{\mu}\left(x^{k_{i}}\right)\right\|=0$. Since $r_{\mu}(\cdot)$ is continuous and $\left\{x^{k_{i}}\right\}$ is a bounded sequence, there exists an accumulation point $\bar{x}$ of $\left\{x^{k_{i}}\right\}$ such that $r_{\mu}(\bar{x})=0$, which implies that $\bar{x}$ solves the $\operatorname{VI}(C, f)$. Replacing $x^{*}$ by $\bar{x}$ in (27), we obtain that the sequence $\left\{\left\|x^{k}-\bar{x}\right\|\right\}$ is also nonincreasing and hence converges. Since $\bar{x}$ is an accumulation point of $\left\{x^{k_{i}}\right\}$, some subsequence of $\left\{\left\|x^{k_{i}}-\bar{x}\right\|\right\}$ converges to zero. This shows that the whole sequence $\left\{\left\|x^{k}-\bar{x}\right\|\right\}$ converges to zero, and hence $\lim _{k \rightarrow \infty} x^{k}=\bar{x}$.

If $\lim _{j \rightarrow \infty} \eta_{k_{j}}=0$, by the search procedure (15), we have

$$
\begin{aligned}
& \left\langle f\left(x^{k_{j}}\right)-f\left(x^{k_{j}}-\eta_{k_{j}} \gamma^{-1} r_{\mu}\left(x^{k_{j}}\right)\right), r_{\mu}\left(x^{k_{j}}\right)\right\rangle \\
& >\sigma\left\|r_{\mu}\left(x^{k_{j}}\right)\right\|^{2} .
\end{aligned}
$$

Since $f$ and $\langle\cdot, \cdot\rangle$ are continuous, we obtain by letting $j \rightarrow$ $\infty$ that $\lim _{j \rightarrow \infty}\left\|r_{\mu}\left(x^{k_{j}}\right)\right\|=0$. Similar discussion obtains the desired result.

\section{The Modified Double Projection Algorithm and Convergence Analysis}

In this section, we present the modified double projection algorithm which finds a solution of the $\operatorname{VI}(C, f)$ which is also a fixed point of a given nonexpansive mapping. Let $S: R^{n} \rightarrow$ $R^{n}$ be a nonexpansive mapping, and denote by $\operatorname{Fix}(S)$ its fixed point set; that is,

$$
\operatorname{Fix}(S)=\left\{x \in R^{n} \mid S(x)=x\right\}
$$

Let $\left\{\alpha_{k}\right\}_{k=0}^{\infty} \subset[c, d]$ for some $c, d \in(0,1)$.

Algorithm 11. Select $x_{0} \in C, \alpha, \beta \geq 0, \sigma>0, \mu \in\left(0, \sigma^{-1}\right)$, $\gamma \in(0,1), \omega \geq \alpha$. Set $k=0$.

Step 1. Compute $r_{\mu}\left(x^{k}\right)$ and $y^{k}=x^{k}-\eta_{k} r_{\mu}\left(x^{k}\right)$, where $\eta_{k}=\gamma^{m_{k}}$ with $m_{k}$ being the smallest nonnegative integer $m$ satisfying the line search (15).

Step 2. Compute

$$
x^{k+1}=\alpha_{k} x^{k}+\left(1-\alpha_{k}\right) S \Pi_{C_{k}}\left(x^{k}\right)
$$

where $C_{k}=C \cap H_{k}$ with $H_{k}=\left\{v \in \mathbb{R}^{n}: h_{k}(v) \leq 0\right\}$ being a halfspace defined by the function (16).

Let $k:=k+1$, and return to Step 1 .

We, next, establish a convergence theorem for Algorithm 11. We assume that the following condition holds.

$$
\operatorname{Fix}(S) \cap \operatorname{SOL}(C, f) \neq \emptyset
$$

We also recall that in $R^{n}$,

$$
\begin{aligned}
\|\lambda x+(1-\lambda) y\|^{2}= & \lambda\|x\|^{2}+(1-\lambda)\|y\|^{2} \\
& -\lambda(1-\lambda)\|x-y\|^{2},
\end{aligned}
$$

for all $x, y \in R^{n}$ and $\lambda \in[0,1]$.

Theorem 12. Suppose that the assumptions of Theorem 10 hold, then any sequence $\left\{x^{k}\right\}$ generalized by Algorithm 11 converges to some point of $\operatorname{Fix}(S) \cap \operatorname{SOL}(C, f)$. 
Proof. Denote $t^{k}=\Pi_{C_{k}}\left(x^{k}\right)$ for all $k \geq 0$. Let $u \in \operatorname{Fix}(S) \cap$ $\operatorname{SOL}(C, f)$. By the definition of $x^{k+1}$, we obtain

$$
\begin{aligned}
\left\|x^{k+1}-u\right\|^{2}= & \left\|\alpha_{k} x^{k}+\left(1-\alpha_{k}\right) S\left(t^{k}\right)-u\right\|^{2} \\
= & \left\|\alpha_{k}\left(x^{k}-u\right)+\left(1-\alpha_{k}\right)\left(S\left(t^{k}\right)-u\right)\right\|^{2} \\
= & \alpha_{k}\left\|x^{k}-u\right\|^{2}+\left(1-\alpha_{k}\right)\left\|S\left(t^{k}\right)-u\right\|^{2} \\
& \quad-\alpha_{k}\left(1-\alpha_{k}\right)\left\|x^{k}-S\left(t^{k}\right)\right\|^{2} \\
\leq & \alpha_{k}\left\|x^{k}-u\right\|^{2}+\left(1-\alpha_{k}\right)\left\|S\left(t^{k}\right)-S(u)\right\|^{2} \\
\leq & \alpha_{k}\left\|x^{k}-u\right\|^{2}+\left(1-\alpha_{k}\right)\left\|t^{k}-u\right\|^{2} \\
\leq & \alpha_{k}\left\|x^{k}-u\right\|^{2}+\left(1-\alpha_{k}\right) \\
& \quad \times\left(\left\|x^{k}-u\right\|^{2}-\left\|t^{k}-x^{k}\right\|^{2}\right) \\
= & \left\|x^{k}-u\right\|^{2}-\left(1-\alpha_{k}\right)\left\|t^{k}-x^{k}\right\|^{2} \\
= & \left\|x^{k}-u\right\|^{2}-\left(1-\alpha_{k}\right) \operatorname{dist}^{2}\left(x^{k}, C_{k}\right),
\end{aligned}
$$

where the third equality follows from (36), the second inequality follows from the nonexpansion of $S$, and the third inequality follows from Lemma 2(2). In (39), using the proof similar to those of Theorem 10, we obtain that $\left\{x^{k}\right\}$ converges to some solution $\bar{x}$ of $\operatorname{VI}(C, f)$. It is now left to show that $\bar{x} \in \operatorname{Fix}(S)$. By (38), we obtain that $\left\{\left\|x^{k}-u\right\|\right\}$ is a convergent sequence; that is, there exists some $\theta>0$, such that

$$
\begin{aligned}
& \lim _{k \rightarrow \infty}\left\|x^{k}-u\right\|=\theta, \\
& \lim _{k \rightarrow \infty}\left\|x^{k}-t^{k}\right\|=0 .
\end{aligned}
$$

Since $S$ is nonexpansive, we obtain

$$
\left\|S\left(t^{k}\right)-u\right\|=\left\|S\left(t^{k}\right)-S(u)\right\| \leq\left\|t^{k}-u\right\| \leq\left\|x^{k}-u\right\|,
$$

which, together with (40), means that

$$
\limsup _{k \rightarrow \infty}\left\|S\left(t^{k}\right)-u\right\| \leq \theta .
$$

Furthermore,

$$
\begin{aligned}
\lim _{k \rightarrow \infty} & \left\|\alpha_{k}\left(x^{k}-u\right)+\left(1-\alpha_{k}\right)\left(S\left(t^{k}\right)-u\right)\right\| \\
= & \lim _{k \rightarrow \infty}\left\|\alpha_{k} x^{k}+\left(1-\alpha_{k}\right) S\left(t^{k}\right)-u\right\| \\
= & \lim _{k \rightarrow \infty}\left\|x^{k+1}-u\right\|=\theta .
\end{aligned}
$$

So applying Lemma 5, we obtain

$$
\lim _{k \rightarrow \infty}\left\|S\left(t^{k}\right)-x^{k}\right\|=0 .
$$

Since

$$
\begin{aligned}
\left\|S\left(x^{k}\right)-x^{k}\right\| & =\left\|S\left(x^{k}\right)-S\left(t^{k}\right)+S\left(t^{k}\right)-x^{k}\right\| \\
& \leq\left\|S\left(x^{k}\right)-S\left(t^{k}\right)\right\|+\left\|S\left(t^{k}\right)-x^{k}\right\| \\
& \leq\left\|x^{k}-t^{k}\right\|+\left\|S\left(t^{k}\right)-x^{k}\right\|,
\end{aligned}
$$

it follows from (41) and (45) that

$$
\lim _{k \rightarrow \infty}\left\|S\left(x^{k}\right)-x^{k}\right\|=0 .
$$

Since $S$ is nonexpansive on $\mathbb{R}^{n}$ and $x^{k}$ converges to $\bar{x}$, we obtain that $\bar{x}-S(\bar{x})=0$, which means that $\bar{x} \in \operatorname{Fix}(S)$. Therefore, the sequence $\left\{x^{k}\right\}$ converges to $\bar{x} \in \operatorname{Fix}(S) \cap$ $\operatorname{SOL}(C, f)$.

Next, we provide a result on the convergence rate of the iterative sequence generated by Algorithm 11. To establish this result, we need the following local error bound condition to hold.

There exist two positive constants $c$ and $\delta$ such that

$$
\begin{array}{r}
\operatorname{dist}(x, \operatorname{Fix}(S) \cap \operatorname{SOL}(C, f)) \leq c\left\|r_{\mu}\left(x^{k}\right)\right\|, \\
\forall x \text { satisfying }\left\|r_{\mu}(x)\right\| \leq \delta .
\end{array}
$$

Theorem 13. In addition to the assumptions of Theorem 10, if $f$ is Lipschitz continuous with modulus $L>0$ and the local error bound condition (48) holds, then there is a constant $\tau>0$ such that for sufficiently large $k$,

$$
\begin{aligned}
& \operatorname{dist}\left(x^{k}, \operatorname{Fix}(S) \cap \operatorname{SOL}(C, f)\right) \\
& \leq \frac{1}{\sqrt{\tau k+\operatorname{dist}^{-2}\left(x^{0}, \operatorname{Fix}(S) \cap \operatorname{SOL}(C, f)\right)}} .
\end{aligned}
$$

Proof. Set $\eta:=\min \left\{1 / 3, L^{-1} \gamma \sigma\right\}$. We first prove that $\eta_{k}>\eta$ for all $k$. By the definition of $\eta_{k}$, we obtain $\eta_{k} \in(0,1]$. If $\eta_{k}=1$, then $\eta_{k}>\eta$ holds. Now, we assume that $\eta_{k}<1$. It follows from $\eta_{k}=\gamma^{m_{k}}$ that $m_{k} \geq 1$. By the construction of $\eta_{k}$, we have

$$
\left\langle f\left(x^{k}\right)-f\left(x^{k}-\eta_{k} \gamma^{-1} r_{\mu}\left(x^{k}\right)\right), r_{\mu}\left(x^{k}\right)\right\rangle>\sigma\left\|r_{\mu}\left(x^{k}\right)\right\|^{2} .
$$

From the Lipschitz continuity of $f$, we have

$$
\begin{aligned}
\sigma\left\|r_{\mu}\left(x^{k}\right)\right\|^{2} & <\left\langle f\left(x^{k}\right)-f\left(x^{k}-\eta_{k} \gamma^{-1} r_{\mu}\left(x^{k}\right)\right), r_{\mu}\left(x^{k}\right)\right\rangle \\
& \leq L \gamma^{-1} \eta_{k}\left\|r_{\mu}\left(x^{k}\right)\right\|^{2} .
\end{aligned}
$$

Thus, $\eta_{k}>L^{-1} \gamma \sigma \geq \eta$. 
TABLE 1: Example 14: Numerical results for initial point $x_{0}=$ $(1,1, \ldots, 1)^{T}$ and $\varepsilon=10^{-4}$.

\begin{tabular}{lcccccc}
\hline \multirow{2}{*}{$\operatorname{Dim}(n)$} & \multicolumn{2}{c}{ Algorithm 6 } & \multicolumn{3}{c}{$[10$, Algorithm 2.1] } & {$[8$, Algorithm 2.2] } \\
& iter & CPU & iter & CPU & iter & CPU \\
\hline 100 & 10 & 0.530 & 11 & 0.577 & 15 & 0.624 \\
200 & 10 & 1.279 & 12 & 1.529 & 15 & 1.778 \\
500 & 11 & 10.905 & 13 & 11.497 & 15 & 13.99 \\
1000 & 12 & 66.63 & 13 & 75.04 & 17 & 104.08 \\
2000 & 12 & 340.9 & 14 & 441.05 & 17 & 610.3 \\
\hline
\end{tabular}

TABle 2: Example 14: Numerical results for initial point $x_{0}=$ $(0,0, \ldots, 0)^{T}$ and $\varepsilon=10^{-4}$.

\begin{tabular}{lcccccc}
\hline \multirow{2}{*}{$\operatorname{Dim}(n)$} & \multicolumn{2}{c}{ Algorithm 6 } & \multicolumn{3}{c}{$[10$, Algorithm 2.1] } & \multicolumn{3}{c}{ 8, Algorithm 2.2] } \\
& iter & CPU & iter & CPU & iter & CPU \\
\hline 100 & 10 & 0.530 & 11 & 0.577 & 15 & 0.593 \\
200 & 10 & 1.185 & 12 & 1.451 & 15 & 1.809 \\
500 & 11 & 10.78 & 13 & 11.06 & 15 & 13.41 \\
1000 & 12 & 65.69 & 13 & 74.16 & 17 & 101.4 \\
2000 & 12 & 341.6 & 14 & 431.4 & 17 & 593.1 \\
\hline
\end{tabular}

Let $u^{*} \in \Pi_{\mathrm{Fix}(S) \cap \operatorname{SOL}(C, f)}\left(x^{k}\right)$. By (29), (39), and (48), we obtain that for sufficiently large $k$,

$$
\begin{aligned}
\operatorname{dist}^{2} & \left(x^{k+1}, \operatorname{Fix}(S) \cap \operatorname{SOL}(C, f)\right) \\
\leq & \left\|x^{k+1}-u^{*}\right\|^{2} \\
\leq & \left\|x^{k}-u^{*}\right\|^{2}-\left(1-\alpha_{k}\right) \operatorname{dist}^{2}\left(x^{k}, C_{k}\right) \\
\leq & \left\|x^{k}-u^{*}\right\|^{2}-M^{-2} \omega^{2} \eta_{k}^{2}(1-\mu \sigma)^{2}\left\|r_{\mu}\left(x^{k}\right)\right\|^{4} \\
\leq & \operatorname{dist}^{2}\left(x^{k}, \operatorname{Fix}(S) \cap \operatorname{SOL}(C, f)\right)-M^{-2} \omega^{2} \eta^{2} \\
& \times(1-\mu \sigma)^{2} c^{-4} \operatorname{dist}^{4}\left(x^{k}, \operatorname{Fix}(S) \cap \operatorname{SOL}(C, f)\right) .
\end{aligned}
$$

Denote $\tau=M^{-2} \omega^{2} \eta^{2}(1-\mu \sigma)^{2} c^{-4}$. Applying Lemma 6 in $[14$, Chapter 2], we have

$$
\begin{aligned}
& \operatorname{dist}\left(x^{k}, \operatorname{Fix}(S) \cap \operatorname{SOL}(C, f)\right) \\
& \leq \frac{1}{\sqrt{\tau k+\operatorname{dist}^{-2}\left(x^{0}, \operatorname{Fix}(S) \cap \operatorname{SOL}(C, f)\right)}} .
\end{aligned}
$$

\section{Numerical Experiments}

In this section, we present some numerical experiments' results to show the effectiveness of the proposed algorithm. The MATLAB codes are run on a notebook computer with Intel Core $2 \mathrm{CPU}$ 2.10 GHZ and RAM 2.00 GM under MATLAB Version 7.0. We compare the performance of our Algorithm 6, [10, Algorithm 2.1] and [8, Algorithm 2.2].
In all tables, we use Dim to mean the dimension of the problem, CPU to represent the CPU total runtime of the computer in seconds, and iter to mean the total iterative number of times. The tolerance $\varepsilon$ means when $\left\|r_{\mu}\left(x_{k}\right)\right\| \leq \varepsilon$, the procedure stops. We choose $\sigma=2.4, \gamma=0.9, \mu=0.26, \alpha$ $=0.04, \beta=0.01$, and $\omega=5$ for our Algorithm 6 in Tables 1 and $2, \sigma=2.4, \gamma=0.9, \mu=0.32, \alpha=0.04, \beta=0.001$, and $\omega=5.3$ as Algorithm 6 in Table 3; $\gamma=0.5, \sigma=4$, and $\mu=0.2$ for Algorithm 2.1 in [10]; $\sigma=0.3, \theta=4$, and $\gamma=0.5$ for Algorithm 2.2 in [8]. The choices of the parameters for the latter two algorithms are what the corresponding references proposed.

Example 14. Consider the affine variational inequality with $C=[0,1]^{n}$ and $f(x)=M x+d$, where

$$
\begin{gathered}
M=\left(\begin{array}{cccccccc}
4 & -2 & 0 & \cdot & \cdot & \cdot & 0 & 0 \\
1 & 4 & -2 & \cdot & \cdot & \cdot & 0 & 0 \\
0 & 1 & 4 & \cdot & \cdot & \cdot & 0 & 0 \\
& & & \vdots & & & \\
0 & 0 & 0 & \cdot & \cdot & \cdot & 4 & -2 \\
0 & 0 & 0 & \cdot & \cdot & \cdot & 1 & 4
\end{array}\right), \\
d=\left(\begin{array}{c}
-1 \\
-1 \\
\vdots \\
-1
\end{array}\right) .
\end{gathered}
$$

This problem was first tested in [15].

From Tables 1 and 2, we can see that our Algorithm 6 is more efficient than Algorithm 2.2 in [8] and Algorithm 2.1 in [10]. It can be observed that the number of iterations of our algorithm is much less than those of $[8,10]$, and the CPU time in our method is shorter than those of $[8,10]$. In addition, for a set of similar problems, it seems that the number of iterations and the CPU time of the above three methods are not very sensitive to starting point. In fact, the initial points of the methods can be chosen randomly.

Example 15. The Kojima-Shindo nonlinear complementarity problem (NCP) (with $n=4$ ) was considered first in [16], where the function $F(x)$ is defined by

$$
f\left(x_{1}, x_{2}, x_{3}, x_{4}\right)=\left(\begin{array}{c}
3 x_{1}^{2}+2 x_{1} x_{2}+2 x_{2}^{2}+x_{3}+3 x_{4}-6 \\
2 x_{1}^{2}+x_{1}+x_{2}^{2}+10 x_{3}+2 x_{4}-2 \\
3 x_{1}^{2}+x_{1} x_{2}+2 x_{2}^{2}+2 x_{3}+9 x_{4}-9 \\
x_{1}^{2}+3 x_{2}^{2}+2 x_{3}+3 x_{4}-3
\end{array}\right) .
$$

Let the feasible set be the simplex $C=\left\{x \in \mathbb{R}_{+}^{n} \mid x_{1}+x_{2}+\right.$ $\left.x_{3}+x_{4}=4\right\}$. 
TABLE 3: Examples 15 and 16.

\begin{tabular}{lccccrrr}
\hline & $\varepsilon$ & \multicolumn{2}{c}{ Algorithm 6 } & \multicolumn{2}{c}{$[10$, Algorithm 2.1] } & \multicolumn{2}{c}{$[8$, Algorithm 2.2] } \\
& & iter & CPU & iter & CPU & iter & CPU \\
\hline Mathiesen & $10^{-4}$ & 22 & 0.281 & 37 & 0.327 & 28 & 0.359 \\
Harnash5 & $10^{-10}$ & 11 & 0.312 & 12 & 0.203 & 22 \\
Harnash10 & $10^{-10}$ & 35 & 0.528 & 41 & 0.312 & 51 & 0.312 \\
\hline
\end{tabular}

Example 16. The example comes from the problem of computing the Cournot-Nash equilibria of $N$-firm noncooperative games. The defining mapping $F: \mathbb{R}^{n} \rightarrow \mathbb{R}^{n}$ is of the form

$$
\begin{array}{r}
f_{i}(q)=c_{i}\left(q_{i}\right)-p\left(\sum_{j=1}^{n} q_{j}\right)-q_{i} p\left(\sum_{j=1}^{n} q_{j}\right), \\
i=1,2, \ldots, n,
\end{array}
$$

where

$$
\begin{gathered}
c_{i}\left(q_{i}\right)=\alpha_{i} q_{i}+\left[\frac{\beta_{i}}{1+\beta_{i}}\right] L_{i}^{-1 / \beta_{i}} q_{i}^{1+1 / \beta_{i}}, \\
p(Q)=5000^{1 / \gamma} Q^{-1 / \gamma},
\end{gathered}
$$

with

$$
Q=\sum_{j=1}^{n} q_{j}
$$

The constants $\alpha_{i}, L_{i}, \beta_{i}$, and $\gamma$ are positive scalars whose data are taken from [17] with $n=5$ and $n=10$.

In Table 3, Mathiesen's test problem is Example 15. We use $x_{0}=(1,1,1,1)^{T}$ as the initial point. The test problem of Harnash 5 and Harnash 10 is Example 16 with $n=5$ and $n=10$, respectively.

From Table 3, we can see that the number of iterations of our algorithm is less than the ones of the methods in $[8,10]$. In Mathiesen's test problem, Table 3 shows that the CPU time in our algorithm is also shorter than those in $[8,10]$.

Tables 1, 2, and 3 show that constructing hyperplane by $f\left(x^{k}\right)$, has a certain significance to change the convergence rate of the iterative sequence.

\section{Conclusion}

In this paper, a class of double projection algorithms for solving pseudomonotone variational inequalities are proposed on the basis of the algorithms in $[8,10]$. The global convergence of the proposed algorithms is proved under the condition that $f$ is continuous and pseudomonotone. The numerical experiments' results show that our algorithms are more efficient if the direction is chosen properly. How to choose a suitable direction for the different kind of variational inequalities problems would be an interesting topic in further research.

In addition, in Algorithm 6, the searching direction is taken as $\alpha \eta_{k} r_{\mu}\left(x^{k}\right)+\beta f\left(x^{k}\right)+\omega \mu f\left(y^{k}\right)$, which is a linear combination of $r_{\mu}\left(x^{k}\right), f\left(x^{k}\right)$ with $f\left(y^{k}\right)$. I wonder whether there exists an other combination for searching direction of projection method. This is also a topic for further research.

\section{Acknowledgment}

This work was supported by the Educational Science Foundation of Chongqing, Chongqing of China (Grant KJ111309).

\section{References}

[1] S. Karamardian, "Complementarity problems over cones with monotone and pseudomonotone maps," Journal of Optimization Theory and Applications, vol. 18, no. 4, pp. 445-454, 1976.

[2] F. Facchinei and J. S. Pang, Finite-Dimensional Variational Inequalities and Complementarity Problems, vol. 1-2 of Springer Series in Operations Research, Springer, New York, NY, USA, 2003.

[3] N. H. Xiu and J. Z. Zhang, "Some recent advances in projectiontype methods for variational inequalities," Journal of Computational and Applied Mathematics, vol. 152, no. 1-2, pp. 559-585, 2003.

[4] G. M. Korpelevič, "An extragradient method for finding saddle points and for other problems," Ėkonomika i Matematicheskie Metody, vol. 12, no. 4, pp. 747-756, 1976.

[5] E. N. Khobotov, "Modification of the extra-gradient method for solving variational inequalities and certain optimization problems," USSR Computational Mathematics and Mathematical Physics, vol. 5, pp. 120-127, 1987.

[6] P. Marcotte, "Application of khobotovs algorithm to variatonal inequalities and network equilibrium problems," Information Systems and Operational Research, vol. 4, pp. 258-270, 1991.

[7] A. N. Iusem and B. F. Svaiter, "A variant of Korpelevich's method for variational inequalities with a new search strategy," Optimization, vol. 42, no. 4, pp. 309-321, 1997.

[8] M. V. Solodov and B. F. Svaiter, "A new projection method for variational inequality problems," SIAM Journal on Control and Optimization, vol. 37, no. 3, pp. 765-776, 1999.

[9] Y. J. Wang, N. H. Xiu, and C. Y. Wang, "Unified framework of extragradient-type methods for pseudomonotone variational inequalities," Journal of Optimization Theory and Applications, vol. 111, no. 3, pp. 641-656, 2001.

[10] Y. R. He, "A new double projection algorithm for variational inequalities," Journal of Computational and Applied Mathematics, vol. 185, no. 1, pp. 166-173, 2006.

[11] N. Nadezhkina and W. Takahashi, "Weak convergence theorem by an extragradient method for nonexpansive mappings and monotone mappings," Journal of Optimization Theory and Applications, vol. 128, no. 1, pp. 191-201, 2006. 
[12] M. A. Noor, Y. J. Wang, and N. H. Xiu, "Projection iterative schemes for general variational inequalities," Journal of Inequalities in Pure and Applied Mathematics, vol. 3, no. 3, article 34, 2002.

[13] Y. J. Wang, N. H. Xiu, and C. Y. Wang, "A new version of extragradient method for variational inequality problems," Computers \& Mathematics with Applications, vol. 42, no. 6-7, pp. 969-979, 2001.

[14] B. T. Polyak, Introduction to Optimization, Translations Series in Mathematics and Engineering, Optimization Software, New York, NY, USA, 1987.

[15] D. Sun, "A class of iterative methods for solving nonlinear projection equations," Journal of Optimization Theory and Applications, vol. 91, no. 1, pp. 123-140, 1996.

[16] J. S. Pang and S. A. Gabriel, "NE/SQP: a robust algorithm for the nonlinear complementarity problem," Mathematical Programming, vol. 60, no. 3, pp. 295-337, 1993.

[17] P. T. Harker, "Accelerating the convergence of the diagonalization and projection algorithms for finite-dimensional variational inequalities," Mathematical Programming, vol. 41, no. 1, pp. 29-59, 1988. 


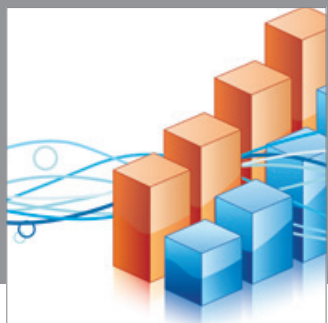

Advances in

Operations Research

mansans

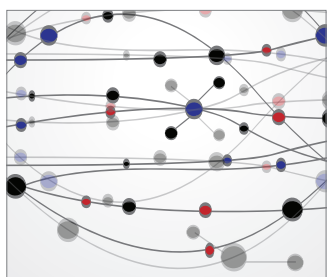

The Scientific World Journal
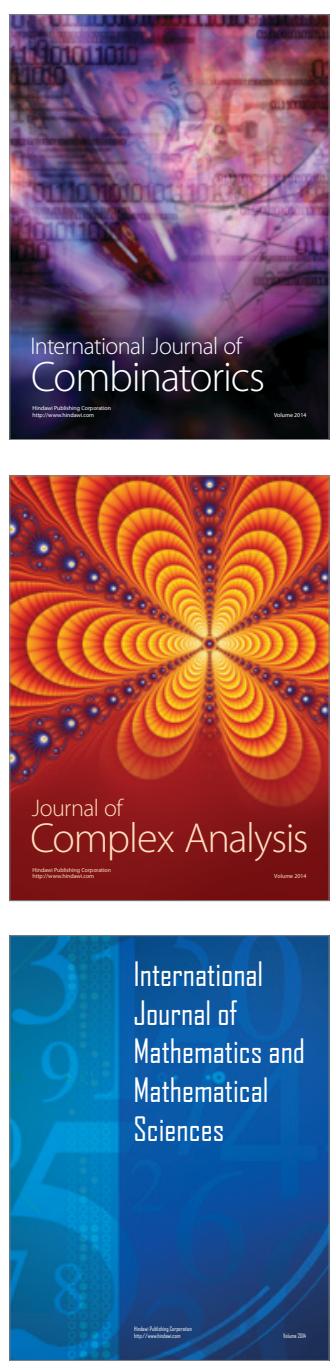
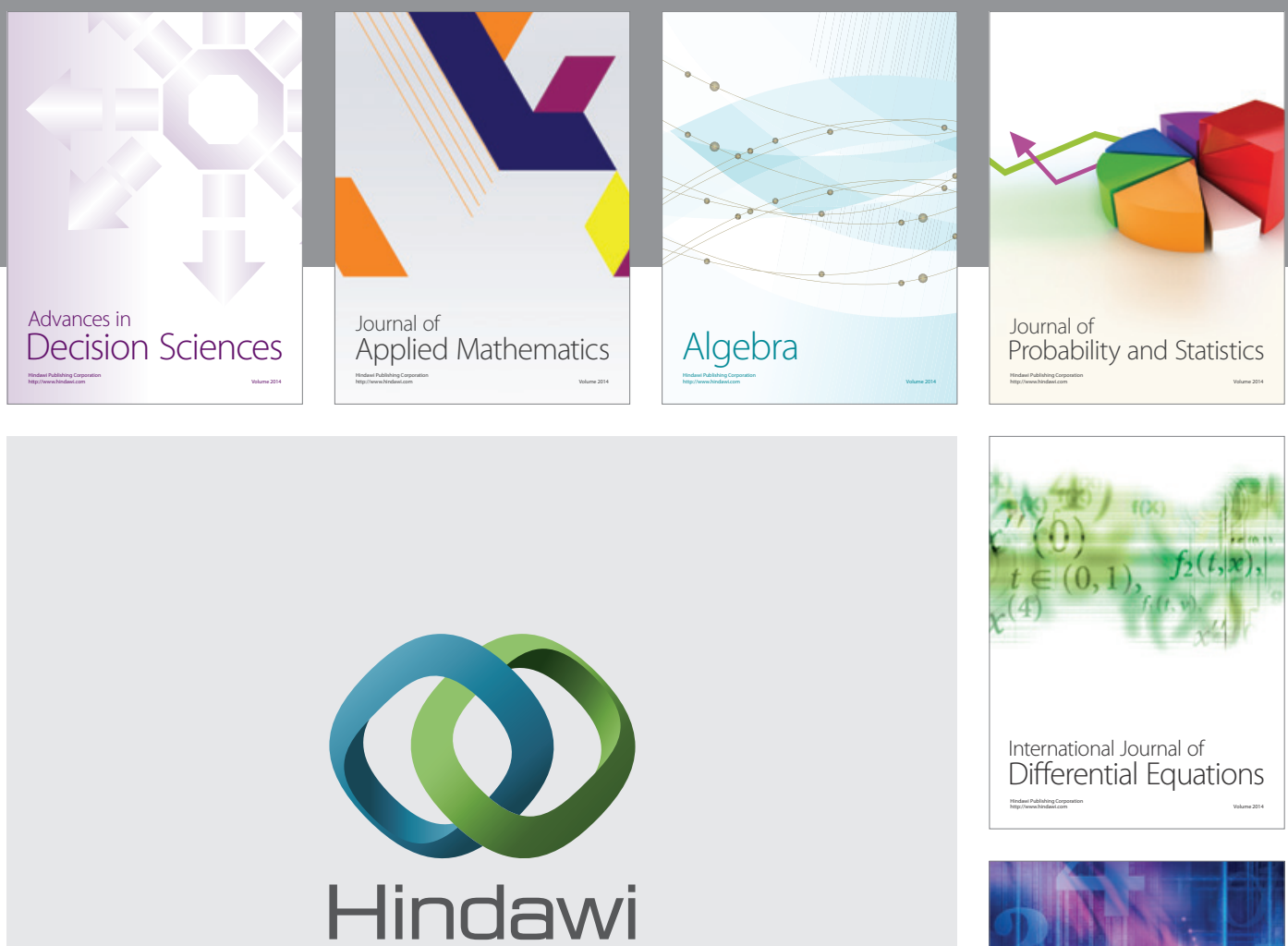

Submit your manuscripts at http://www.hindawi.com
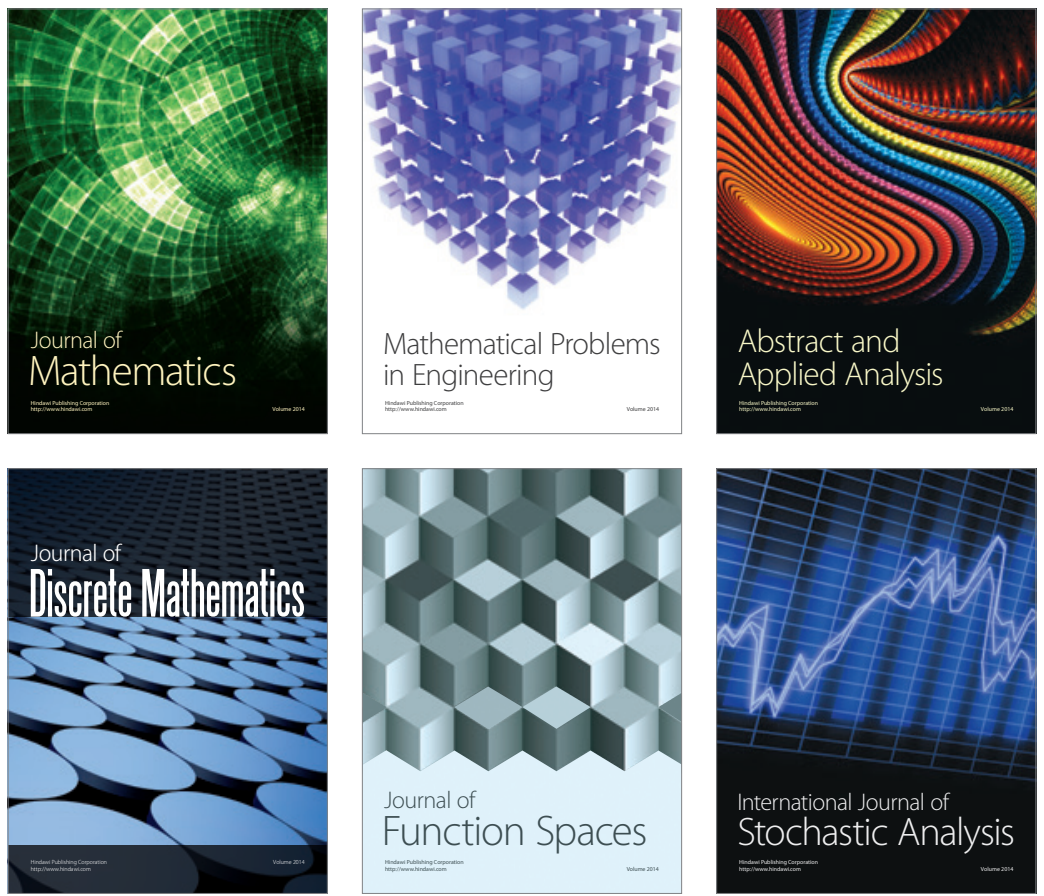

Journal of

Function Spaces

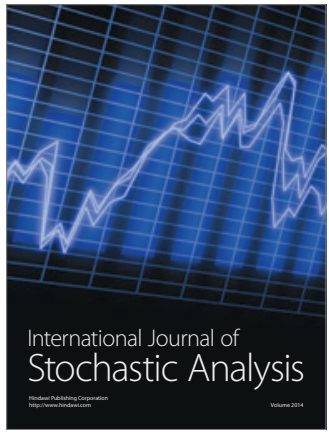

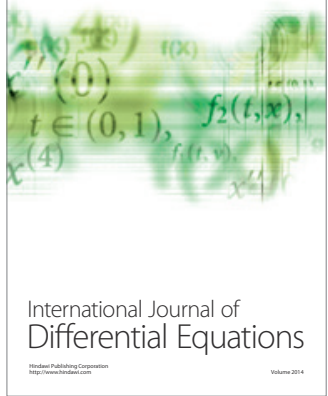
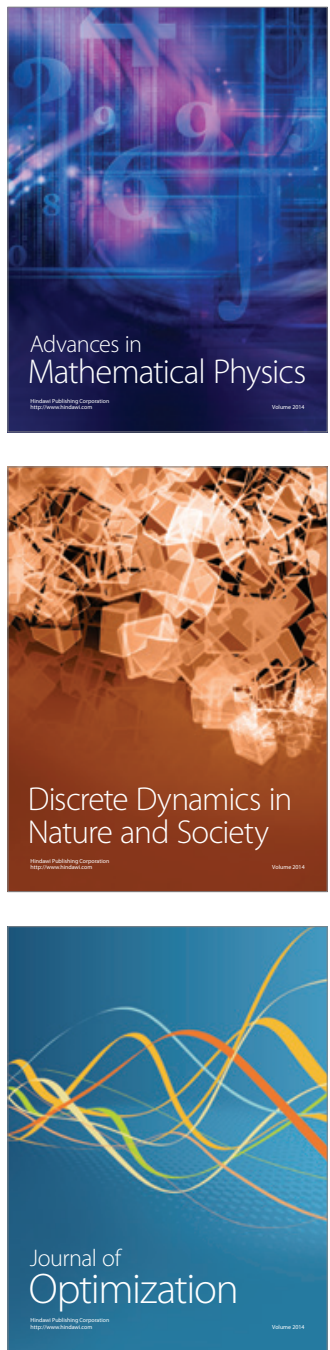